\title{
VAN DER POL'S OSCILLATOR UNDER THE PARAMETRIC AND FORCED EXCITATIONS
}

\author{
NGUYEN VAN DAO \\ Vietnam National University, Hanoi \\ Nguyen Van Dinh, Tran Kim Chi \\ Vietnam Academy of Sciences, Hanoi \\ (This paper has been published in: \\ Український Математичний Журнал 2007, Том 59, $\mathrm{N}^{0} 2$ )
}

\begin{abstract}
Van der Pol's oscillator under parametric and forced excitations is studied. The case where the system contains a small parametrer being quasilinear and the general case (without assumption on the smallness of nonlinear terms and perturbations) are studied. In the first case, equations of the first approximation are obtained by means of the Krylov-Bogoliubov-Mitropolskii technique, their averaging is performed, frequencyamplitude and resonance curves are studied, on the stability of the given system is considered. In the second case, the possibility of chaotic behavior in a deterministic system of oscillator type is shown.
\end{abstract}

\section{INTRODUCTION}

It is well-known that there always exists an interaction of some kind between nonlinear oscillating systems. N. Minorsky stated that "Perhaps the whole theory of nonlinear oscillations could be formed on the basis of interaction" [3]. Different interesting cases of interaction have been investigated by us and published in the monograph [3], using the effective asymptotic method of nonlinear mechanics created by Krylov N. M., Bogoliubov N. N. and Mitropolskii Yu. A.

The present paper introduces our research on the behaviour of a Van der Pol's oscillator under the parametric and forced excitations. The dynamic system under consideration is described by an ordinary nonlinear differential equation of type (2.1). The section 1 is devoted to the case of small parameters. The amplitudes of nonlinear deterministic oscillations and their stability are studied. Analytical calculations in combination with a computer are used to obtain amplitude curves, which show a very complicated form in Figs. $1-4$. In the section 2 we study the chaotic phenomenon occurring in the system described by equation (2.1) without assumption on the smallness of the parameters.

As known, the fundamental characteristic of a chaotic system is its sensitivity to the initial conditions. The diagnostic tool used in this work is the Liapunov exponents. The positiveness of the largest Liapunov exponent will help us to define the values of parameters with which the chaotic motions are occurred. Chaotic attractors and associated power spectra will be presented. 


\section{THE CASE OF SMALL PARAMETERS}

In this section, let us consider the case when the parameters are small. The opposite case will be investigated in the next section. The smallness of parameters is characterized by introducing a small positive parameter $\varepsilon$. For this case the asymptotic method of Krylov - Bogoliubov - Mitropolskii (K-B-M) [1, 2] is used to seek the approximate solutions and to study their stability.

\subsection{The differential equation of oscillation and its stationary solution}

The system under consideration is described by the equation

$$
\ddot{x}+\omega^{2} x=\varepsilon\left\{\Delta x-\gamma x^{3}+h\left(1-k x^{2}\right) \dot{x}+2 p x \cos 2 \omega t+e \cos (\omega t+\sigma)\right\},
$$

where $h>0$ and $k>0$ are coefficients characterizing the self-excitation of a pure Van der Pol's oscillator, $2 p>0$ is the intensity of the parametric excitation, $e>0$ is the intensity of the forced excitation and $\sigma, 0 \leqslant \sigma \leqslant 2 \pi$ is the phase shift between the parametric and forced excitations. Bellow, two subcases will be investigated separately for a weak parametric excitation when $p^{2}<h^{2}$ and for a strong parametric excitation when $p^{2}>h^{2}$. The solution of (2.1) is found in the form

$$
x=a \cos \psi, \quad \dot{x}=-a \omega \sin \psi, \quad \psi=\omega t+\theta,
$$

where $a$ and $\theta$ are new variables, which satisfy the following equations in the standard form

$$
\begin{aligned}
& \dot{a}=-\frac{\varepsilon}{\omega_{\varepsilon}}\left\{\Delta x-\gamma x^{3}+h\left(1-k x^{2}\right) \dot{x}+2 p x \cos 2 \omega t+e \cos (\omega t+\sigma)\right\} \sin \psi, \\
& \dot{\theta}=-\frac{{ }^{2}}{a \omega}\left\{\Delta x-\gamma x^{3}+h\left(1-k x^{2}\right) \dot{x}+2 p x \cos 2 \omega t+e \cos (\omega t+\sigma)\right\} \cos \psi .
\end{aligned}
$$

Following the K-B-M method, in the first approximation these equations can be replaced by averaged ones

$$
\begin{aligned}
& \dot{a}=-\frac{\varepsilon}{2 \omega} f_{0}=-\frac{\varepsilon}{2 \omega}\left\{h \omega\left(\frac{k a^{2}}{4}-1\right) a+p a \sin 2 \theta+e \sin (\theta-\sigma)\right\}, \\
& a \dot{\theta}=-\frac{\varepsilon}{2 \omega} g_{0}=-\frac{\varepsilon}{2 \omega}\left\{\Delta a-\frac{3}{4} \gamma a^{3}+p a \cos 2 \theta+e \cos (\theta-\sigma)\right\} .
\end{aligned}
$$

The amplitude $a$ and phase $\theta$ of stationary oscillations are determined from the equations $\dot{a}=\dot{\theta}=0$ :

$$
\begin{aligned}
& f_{0}=h \omega\left(\frac{k a^{2}}{4}-1\right) a+p a \sin 2 \theta+e \sin (\theta-\sigma)=0, \\
& g_{0}=\Delta a-\frac{3}{4} \gamma a^{3}+p a \cos 2 \theta+e \cos (\theta-\sigma)=0 .
\end{aligned}
$$

These equations are equivalent to

$$
\begin{aligned}
& f=f_{0} \cos \theta-g_{0} \sin \theta=\left(p-\Delta+\frac{3}{4} \gamma a^{2}\right) a \sin \theta+h \omega\left(\frac{k a^{2}}{4}-1\right) a \cos \theta-e \sin \sigma=0, \\
& g=f_{0} \sin \theta+g_{0} \cos \theta=h \omega\left(\frac{k a^{2}}{4}-1\right) a \sin \theta+\left(p+\Delta-\frac{3}{4} \gamma a^{2}\right) a \cos \theta+e \cos \sigma=0,
\end{aligned}
$$

or

$$
\begin{aligned}
& f=A \sin \theta+B \cos \theta-E=0, \\
& g=G \sin \theta+H \cos \theta-K=0,
\end{aligned}
$$


where

$$
\begin{aligned}
& A=\left(p-\Delta+\frac{3}{4} \gamma a^{2}\right) a, \quad B=h \omega\left(\frac{k a^{2}}{4}-1\right) a, \quad E=e \sin \sigma, \\
& G=h \omega\left(\frac{k a^{2}}{4}-1\right) a, \quad H=\left(p+\Delta-\frac{3}{4} \gamma a^{2}\right) a, \quad K=-e \cos \sigma .
\end{aligned}
$$

\subsection{The amplitude-frequency relationship and resonance curve}

The characteristic determinants of equations (2.7) are

$$
\begin{aligned}
& D=\left|\begin{array}{cc}
A & B \\
G & H
\end{array}\right|=\left|\begin{array}{cc}
\left.p-\Delta+\frac{3}{4} \gamma a^{2}\right) a & h \omega\left(\frac{k a^{2}}{4}-1\right) a \\
h \omega\left(\frac{k a^{2}}{4}-1\right) a & \left(p+\Delta-\frac{3}{4} \gamma a^{2}\right) a
\end{array}\right|= \\
& =a^{2}\left\{\left(p+\Delta-\frac{3}{4} \gamma a^{2}\right)\left(p-\Delta+\frac{3}{4} \gamma a^{2}\right)-h^{2} \omega^{2}\left(\frac{k a^{2}}{4}-1\right)^{2}\right\} \text {, } \\
& D_{1}=\left|\begin{array}{ll}
E & B \\
K & H
\end{array}\right|=\left|\begin{array}{ll}
e \sin \sigma & h \omega\left(\frac{k a^{2}}{4}-1\right) a \\
-e \cos \sigma & \left(p+\Delta-\frac{3}{4} \gamma a^{2}\right) a
\end{array}\right|= \\
& =a e\left\{\left(p+\Delta-\frac{3}{4} \gamma a^{2}\right) \sin \sigma+h \omega\left(\frac{k a^{2}}{4}-1\right) \cos \sigma\right\} \text {, } \\
& D_{2}=\left|\begin{array}{cc}
A & E \\
G & K
\end{array}\right|=\left|\begin{array}{cc}
\left(p-\Delta+\frac{3}{4} \gamma a^{2}\right) a & e \sin \sigma \\
h \omega\left(\frac{k a^{2}}{4}-1\right) a & -e \cos \sigma
\end{array}\right|= \\
& =-a e\left\{\left(p-\Delta+\frac{3}{4} \gamma a^{2}\right) \cos \sigma+h \omega\left(\frac{k a^{2}}{4}-1\right) \sin \sigma\right\} .
\end{aligned}
$$

Below, in the $(\Delta, a)$-plane we identify the regular region in which the characteristic determinant $D$ is nonzero and the critical region in which $D$ is identically zero.

By solving equations (2.7) relatively to $\sin \theta$ and $\cos \theta$ and eliminating the phase $\theta$ we obtain the amplitude-frequency relationship

$$
\begin{gathered}
W(\Delta, a)=D_{1}^{2}+D_{2}^{2}-D^{2}= \\
=a^{2} e^{2}\left\{\left(p+\Delta-\frac{3}{4} \gamma a^{2}\right) \sin \sigma+h \omega\left(\frac{k a^{2}}{4}-1\right) \cos \sigma\right\}^{2}+ \\
+a^{2} e^{2}\left\{\left(p-\Delta+\frac{3}{4} \gamma a^{2}\right) \cos \sigma+h \omega\left(\frac{k a^{2}}{4}-1\right) \sin \sigma\right\}^{2}- \\
-a^{4}\left\{\left(p-\Delta+\frac{3}{4} \gamma a^{2}\right)\left(p+\Delta-\frac{3}{4} \gamma a^{2}\right)-h^{2} \omega^{2}\left(\frac{k a^{2}}{4}-1\right)^{2}\right\}^{2}=0 .
\end{gathered}
$$

The regular part $C_{1}$ of the resonance curve satisfies (2.10) and lies in the regular region, where $D \neq 0$.

The critical part $C_{2}$ of the resonance curve lies in the critical region, where

$$
D=0 \text { or } p^{2}-\left(\Delta-\frac{3}{4} \gamma a^{2}\right)^{2}-h^{2} \omega^{2}\left(\frac{k a^{2}}{4}-1\right)^{2}=0
$$

and satisfies: 
+ The compatibility conditions

$$
\begin{aligned}
& D_{1}=0 \text { or }\left(p+\Delta-\frac{3}{4} \gamma a^{2}\right) \sin \sigma+h \omega\left(\frac{k a^{2}}{4}-1\right) \cos \sigma=0, \\
& D_{2}=0 \text { or }\left(p-\Delta+\frac{3}{4} \gamma a^{2}\right) \cos \sigma+h \omega\left(\frac{k a^{2}}{4}-1\right) \sin \sigma=0 .
\end{aligned}
$$

+ The trigonometrical conditions

$$
\begin{aligned}
& A^{2}+B^{2} \geqslant E^{2} \text { or } a^{2}\left\{\left(p-\Delta+\frac{3}{4} \gamma a^{2}\right)^{2}+h^{2} \omega^{2}\left(\frac{k a^{2}}{4}-1\right)^{2}\right\} \geqslant e^{2} \sin ^{2} \sigma, \\
& G^{2}+H^{2} \geqslant K^{2} \text { or } a^{2}\left\{\left(p+\Delta-\frac{3}{4} \gamma a^{2}\right)^{2}+h^{2} \omega^{2}\left(\frac{k a^{2}}{4}-1\right)^{2}\right\} \geqslant e^{2} \cos ^{2} \sigma .
\end{aligned}
$$

It is easy to see that the critical region is the resonance curve of Van der Pol's oscillator under the action of the parametric excitation without the forced excitation $(e=0)$. For a weak parametric excitation $\left(p^{2}<h^{2}\right)$, the resonance curve is an oval encircling the-point $A_{0}\left(\Delta=\frac{3}{4} \gamma a^{2}, a^{2}=a_{0}^{2}=\frac{4}{k}\right)$ which is the representative point of the self-oscillation of Van der Pol's oscillator. This oval lies completely above the abscissa axis $\Delta$. When the parametric excitation is strong enough $\left(p^{2}>h^{2}\right)$, the critical oval enlarges and cuts the abscissa axis.

From the compatibility conditions it follows that

$$
\Delta=p \cos 2 \sigma+\frac{3}{4} \gamma a^{2}, \quad h \omega\left(\frac{k a^{2}}{4}-1\right)=-p \sin 2 \sigma .
$$

Hence, the compatibility point has coordinates

$$
\Delta=\Delta_{*}=p \cos 2 \sigma+\frac{3}{4} \gamma a_{*}^{2}, \quad a^{2}=a_{*}^{2}=a_{0}^{2}\left\{1-\frac{p \sin 2 \sigma}{h \sqrt{1+p \cos 2 \sigma}}\right\}
$$

The existence condition of the compatibility point is

$$
a_{*}^{2}>0 \quad \text { or } \frac{p \sin 2 \sigma}{h \sqrt{1+p \cos 2 \sigma}}<1
$$

Obviously, this condition is satisfied if

$$
\sin 2 \sigma<0 \text { i.e. } \frac{\pi}{2}<\sigma<\pi \text { or } \frac{3 \pi}{2}<\sigma<2 \pi .
$$

In the case

$$
\sin 2 \sigma \geqslant 0 \text { i.e. } 0 \leqslant \sigma \leqslant \frac{\pi}{2} \text { or } \pi \leqslant \sigma \leqslant \frac{3 \pi}{2}
$$

the condition (2.13) can be transformed into

$$
\Lambda(\cos 2 \sigma)=p^{2} \cos ^{2} 2 \sigma+p h^{2} \cos 2 \sigma+h^{2}-p^{2} \geqslant 0 .
$$

The left-handside of (2.15) is a trinomial of $\cos 2 \sigma \in[-1,1]$ with the discriminant

$$
\Gamma=p^{2}\left(h^{4}-4 h^{2}+4 p^{4}\right) .
$$




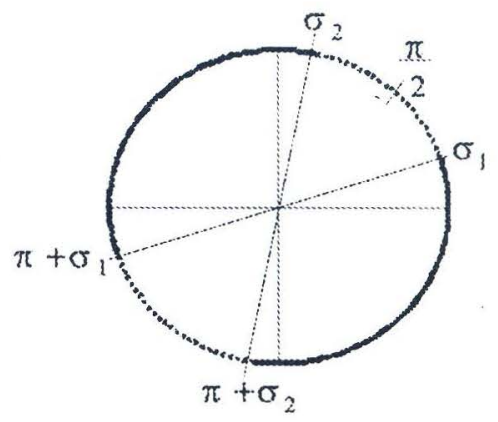

Fig. 1. The heavy arcs give the values $\sigma$ with which the compatibility point $I_{*}$ exists

If $h>2-2 \sqrt{1-p}$ (the case $h^{2}>2+2 \sqrt{1-p}$ is not considered here) then $\Gamma<0$ and the trinomial $\Lambda(\cos 2 \sigma)$ always has the same positive sign as its first coefficient, and the condition (2.15) is satisfied with all values of $\sigma$ in the interval (2.14).

If $h^{2} \leqslant 2-2 \sqrt{1-p}$, then $\Gamma \geqslant 0$ and the trinomial $\Lambda(\cos 2 \sigma)$ has either two simple roots or a double root. The simple roots $\cos 2 \sigma_{1,2}$ are

$$
\cos 2 \sigma_{1,2}=\frac{1}{2 p^{2}}\left(-p h^{2} \pm \sqrt{\Gamma}\right) .
$$

It is noted that $\Lambda(1)=h^{2}(1+p)>0, \Lambda(-1)=h^{2}(1-p),(p=0(\varepsilon))$ and the numerical average of two roots: $\frac{S}{2}=-\frac{p h^{2}}{2 p^{2}}$ satisfy $-1<\frac{S}{2}<1$. Hence, two roots $(2.16)$ lie in the interval $[-1,1]$. The condition (2.15) leads to

$$
\cos 2 \sigma \leqslant \cos 2 \sigma_{2} \quad \text { or } \quad \cos 2 \sigma \geqslant \cos 2 \sigma_{1} .
$$

Combining (2.17) with (2.14) we obtain

$$
0 \leqslant \sigma \leqslant \sigma_{1} \text { or } \sigma_{2} \leqslant \sigma \leqslant \frac{\pi}{2}, \text { or } \pi \leqslant \sigma \leqslant \pi+\sigma_{1} \text { or } \pi+\sigma_{2} \leqslant \sigma \leqslant \frac{3 \pi}{2} .
$$

In summary, we have

+ If $h^{2}>2-2 \sqrt{1-p^{2}}$, then the compatibility point $I_{*}$ exists for every $\sigma$.

+ If $h^{2} \leqslant 2-2 \sqrt{1-p^{2}}$, then the compatibility point $I_{*}$ exists only for

$$
0 \leqslant \sigma \leqslant \sigma_{1} \text { or } \sigma_{2} \leqslant \sigma \leqslant \pi+\sigma_{1} \text { or } \pi+\sigma_{2} \leqslant \sigma \leqslant 2 \pi \text {. }
$$

In Fig. 1 the heavy arcs give the values $\sigma$ with which the compatibility point $I_{*}$ exists when $h^{2} \leqslant 2-2 \sqrt{1-p^{2}}$. Since $2-2 \sqrt{1-p^{2}}$ is approximately equal to $p^{2}$, then

+ If $p^{2}<h^{2}$, i.e. when the parametric excitation is weak in comparison with selfexcitation, the critical oval $D=0$ lies completely above the abscissa axis $\Delta$. The critical point $I_{*}$ always exists.

+ If $p^{2} \geqslant h^{2}$, i.e. when the parametric excitation is strong enough, the critical oval $D=0$ cuts the abscissa axis $\Delta$. The critical point $I_{*}$ exists only with the values of $\sigma$ lying in the interval (2.18). 
Verifying the trigonometrical conditions by substituting (2.12) into (2.11) we obtain

$$
4 p^{2} a_{*}^{2} \sin ^{2} \sigma \geqslant e^{2} \sin ^{2} \sigma, \quad 4 p^{2} a_{*}^{2} \cos ^{2} \sigma \geqslant e^{2} \cos ^{2} \sigma .
$$

Because the right-hand sides of (2.19) are not equal to zero simultaneously, from (2.19) we find

$$
4 p^{2} a_{*}^{2} \geqslant e^{2} \quad \text { or } \quad a_{*}^{2} \geqslant \frac{e^{2}}{4 p^{2}} .
$$

Hence, the compatibility point $I_{*}$ is only a critical point when the amplitude $a$ is large enough, i.e. when the forced excitation is still not too strong in comparison with the parametric one.

\subsection{Forms of resonance curves}

To identify the forms of resonance curves we give in advance the values $h k$, then for each chosen value $p$ we change $e$ and $\sigma$ to have the resonance curves. For example, with $h=0.1, k=4, \omega=1$, the self-excited oscillation of Van der Pol's original system has an amplitude $a_{0}^{2}=1$ and is represented by the point $A_{0}\left(\Delta=\frac{3}{4} \gamma, a_{0}^{2}=1\right)$.

a) The case of weak parametric excitation $\left(p^{2}<h^{2}\right)$

As it is known, in this case the critical oval $D=0$, i.e. the resonance curve of Van der Pol's oscillator under the parametric excitation, runs around the point $A_{0}$ and lies entirely above the abscissa axis $\Delta$. We take $p=0.05$, and $\sigma=0$. For a weak forced excitation, i.e. when $e$ is small enough, the condition (2.20) is satisfied and the critical point $I_{*}$ with coordinates $\left(\Delta_{*}=p+\frac{3}{4} \gamma, a_{*}^{2}=a_{0}^{2}=1\right)$ exists. For enough strong forced excitation, i.e. when $e$ is large enough, point $I_{*}$ is only a trivial compatibility point which does not belong to the resonance curve.

In the Fig. 2 the resonance curves ' 0 ', ' 1 ', ' 2 ', ' 3 ', ' 4 ', ' 5 ' correspond to the linear case $\gamma=0$, for $e=0 ; 0.015 ; 0.017 ; 0.050 ; 0.100 ; 0.120$, respectively. The curve ' 0 ' is a critical oval. The curve ' 1 ' has two branches: branch $C$ ' lies near abscissa axis, branch $C^{\prime \prime}$ lies higher and consists of two cycles, one of them $C_{1}^{\prime \prime}$ is outside and the other $C_{2}^{\prime \prime}$ is inside the critical oval. These cycles are connected to one another at the critical node $I_{*}$ on the critical oval.

Increasing the forced excitation (e), the lower branch $C^{\prime}$ moves up. The inner loop $C_{2}^{\prime \prime}$ of the upper branch is pressed while the outer loop $C_{1}^{\prime \prime}$ is expanded, but both loops are tied at the node $I_{*}$. For $e \approx 0.0177$, the lower branch $C^{\prime}$ is connected with the outer loop $C_{1}^{\prime \prime}$ at the node $J$ and we have the curve '2', where $J$ is a singular point belonging to the regular region $D \neq 0$. For $e$ larger than 0.0177 , the singular point $J$ disappears. Then the lower branch and the outer loop are unified into one branch which lies outside the critical oval. We have the resonance curve ' 3 '. Increasing $e$ further, the inner loop $C_{2}^{\prime \prime}$ continues to be pressed into $I_{*}$ and disappears when $e=0.1$ (see the resonance curve ' 4 '). At this moment $I_{*}$ is a returning point. The curve ' 5 ' corresponds to a very large value of forced excitation; the point $I_{*}$ is a trivial compatibility point which lies outside the resonance curve and does not belong to it.

Fig. 3a, Fig. 3b show the resonances curves in the nonlinear case $\gamma x^{3}, \gamma \neq 0$ with $\gamma=-0.1$ (a) and $\gamma=0.1$ (b). The curves ' 1 ', ' 3 ', ' 5 ' in these figures have the same values of parameters (except $\gamma$ ) of the curves '1', '3', '5' in Fig. 2. 


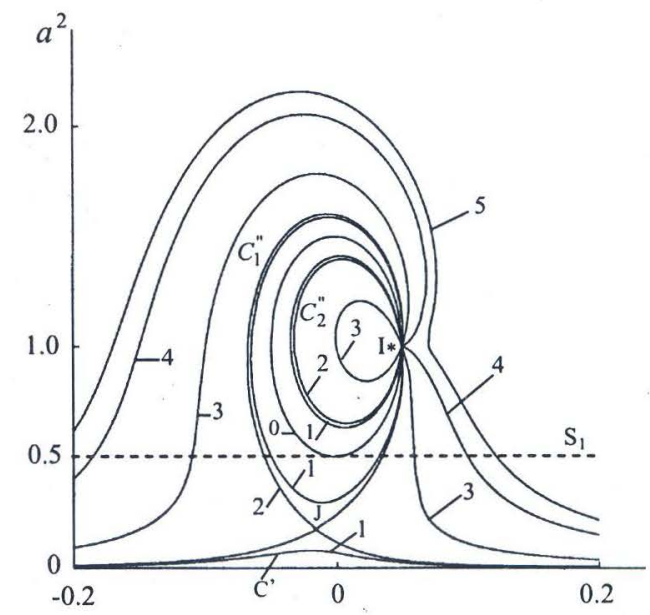

Fig. 2. Resonance curves for $\gamma=0, e=0$ (curve 0), $e=0.115$ (curve 1) $e=0.0177$ (curve 2), $e=0.050$ (curve 3), $e=0.100$ (curve 4), $e=0.120$ (curve 5)
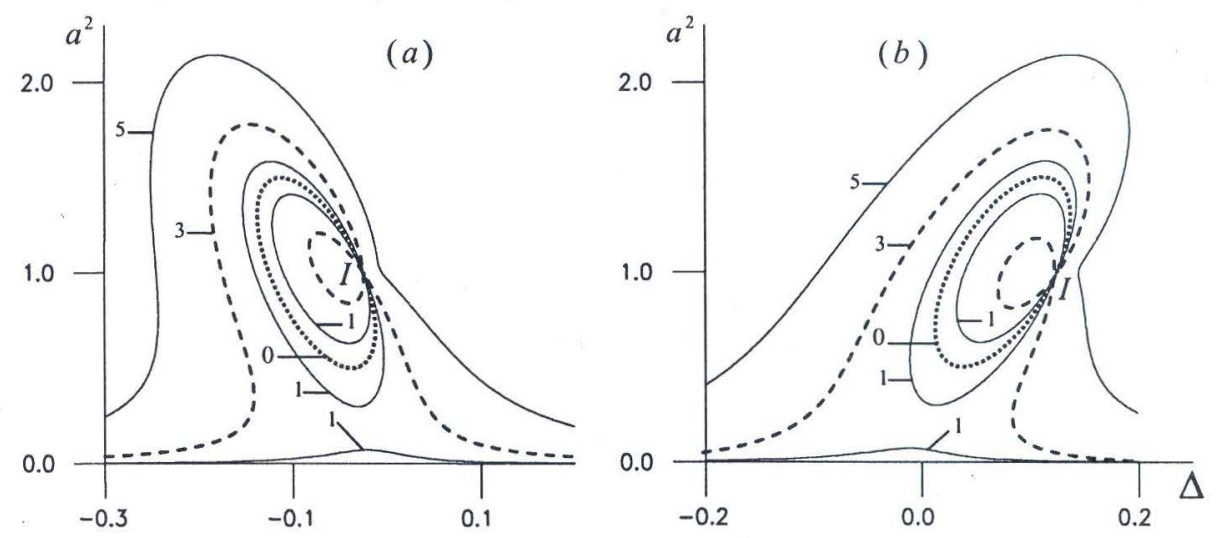

Fig. 3. Resonance curves for $\gamma=0.1$ (a), $\gamma=0.1$ (b), $\sigma=0, e=0$ (curve 0), $e=0.015$ (curve 1 ),$e=0.050$ (curve 3 ), $e=0.12$ (curve 5)

With the negative value of $\gamma$ (see Fig. 3a) resonance curves lean toward the left in comparison with the case $\gamma=0$ (Fig. 2). Otherwise, resonance curves lean toward the right for the positive value of $\gamma$ (see Fig. 3b). This situation is common for nonlinear Duffing's systems.

b) The case of strong parametric excitation $\left(p^{2}>h^{2}\right)$.

As before we take $h=0.1, k=4$ but $p=0.12$. In this case the critical oval ' 0 ' is enlarged and cuts the abscissa axis $\Delta$.

In Fig. 4a $(\gamma=0)$ and Fig. $4 \mathrm{~b}(\gamma=0.1)$, the resonance curves ' 1 ' correspond to $\sigma=0, e=0.06$. The curve ' 1 ' has a cycle lying inside the critical oval and is connected with the outside branch by the critical point $I_{*}$. If only $e$ increases, the inside cycle is tied and then disappears. The critical point $I_{*}$ first becomes a returning point and then an isolated trivial compatibility point. The resonance curve is the only outside branch which is moving up. 

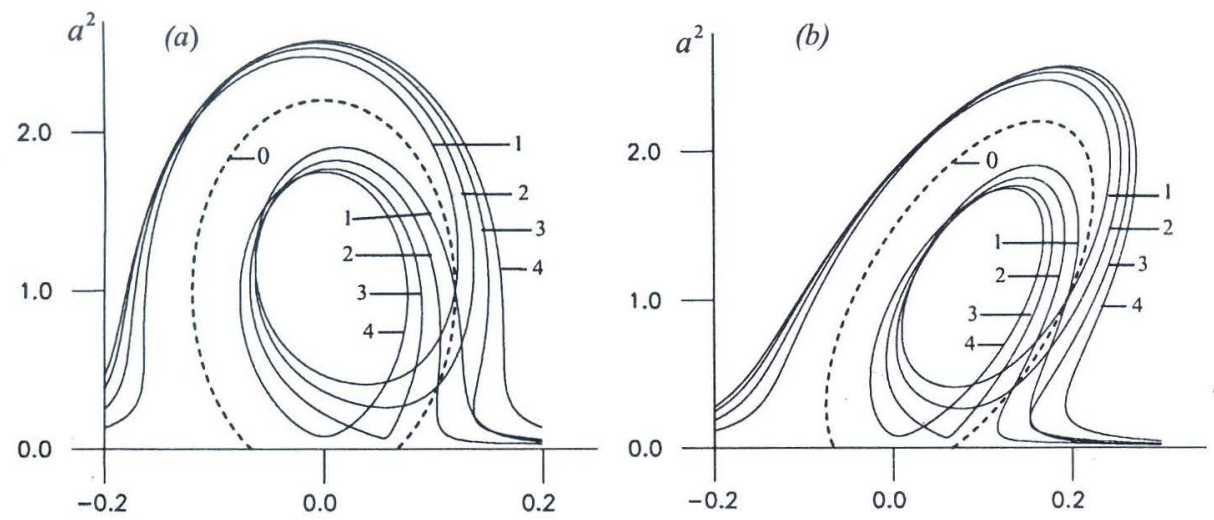

Fig. 4. Resonance curves for $\gamma=0$ (a), $\gamma=0.1(\mathrm{~b})$, and $\sigma=0$ (curves 1), $\sigma=$ $\frac{\pi}{12}($ curves 2$), \sigma=\frac{\pi}{6}$ (curves 3$), \sigma=\frac{\pi}{4}$ (curves 4$)$

Changing $\sigma$, the critical point moves along the critical oval ' 0 '. In Fig. $4 \mathrm{a}$, Fig. $4 \mathrm{~b}$ the resonance curves ' 2 ', ' 3 ', ' 4 ' correspond to the values $\gamma=0(a), \gamma=0.1(b)$ and $\sigma=\frac{\pi}{12} ; \frac{\pi}{6}$; $\frac{\pi}{4}$ respectively. We see that, when $\sigma$ increases the critical point moves down, the critical point $I_{*}$ becomes a returning one and then disappears. Then the resonance curves separate into a cycle lying inside the oval ' 0 ' and a branch lying outside this oval (curves ' 3 ', '4').

\subsection{Stability conditions}

To have the stability condition we use the variational equations by putting in (2.4) $a=a_{0}+\delta a, \theta=\theta_{0}+\delta \theta$ and neglecting the terms of higher degrees with respect to $\delta a$ and $\delta \theta$

$$
\begin{aligned}
\frac{d}{d t}(\delta a) & =-\frac{\varepsilon}{2 \omega}\left(\frac{\partial f_{0}}{\partial a}\right)_{0} \delta a-\frac{\varepsilon}{2 \omega}\left(\frac{\partial f_{0}}{\partial \theta}\right)_{0} \delta \theta, \\
a_{0} \frac{d}{d t}(\delta \theta) & =-\frac{\varepsilon}{2 \omega}\left(\frac{\partial g_{0}}{\partial a}\right)_{0} \delta a-\frac{\varepsilon}{2 \omega}\left(\frac{\partial g_{0}}{\partial \theta}\right)_{0} \delta \theta,
\end{aligned}
$$

where $a_{0}, \theta_{0}$ are stationary values of the amplitude $a$ and phase $\theta$ - the roots of equations (2.5). The characteristic equation of the system (2.21) is

$a_{0} \rho^{2}+\frac{\varepsilon}{2 \omega}\left\{a_{0}\left(\frac{\partial f_{0}}{\partial a}\right)_{0}+\left(\frac{\partial g_{0}}{\partial \theta}\right)_{0}\right\} \rho+\frac{\varepsilon^{2}}{4 \omega^{2}}\left\{\left(\frac{\partial f_{0}}{\partial a}\right)_{0}\left(\frac{\partial g_{0}}{\partial \theta}\right)_{0}-\left(\frac{\partial f_{0}}{\partial \theta}\right)_{0}\left(\frac{\partial g_{0}}{\partial a}\right)_{0}\right\}=0$.

Hence, the stability conditions for stationary solutions $a_{0}, \theta_{0}$ is

$$
\begin{aligned}
S_{1}= & a_{0}\left(\frac{\partial f_{0}}{\partial a}\right)_{0}+\left(\frac{\partial g_{0}}{\partial \theta}\right)_{0}=a_{0}\left\{h \omega\left(\frac{k a_{0}^{2}}{4}-1\right)+\frac{h k}{4} \omega a_{0}^{2}+p \sin 2 \theta_{0}\right\}- \\
& -\left\{2 p a_{0} \sin 2 \theta_{0}+e \sin \left(\theta_{0}-\sigma\right)\right\}>0 .
\end{aligned}
$$

From the first equation of (2.5) we find $\sin 2 \theta_{0}$, then by substituting into (2.22) we get

$$
S_{1}=h \omega a_{0}\left(k a_{0}^{2}-2\right)>0 \text { or } a_{0}^{2}>\frac{2}{k} .
$$

This condition means that only the oscillations with large amplitudes may be stable. 
The second stability condition still has the abbreviated form $[1,4]$

$$
S_{2}=\frac{a_{0}}{D} \frac{\partial W\left(\Delta, a_{0}^{2}\right)}{\partial a_{0}^{2}}>0
$$

The curves $D\left(\Delta, a_{0}^{2}\right)=0$ and $W\left(\Delta, a_{0}^{2}\right)=0$ divide the plane $P\left(\Delta, a_{0}^{2}\right)$ into regions. In each region the functions $D\left(\Delta, a_{0}^{2}\right)$ and $W\left(\Delta, a_{0}^{2}\right)$ have a determined sign. Moving upwards along a straight line, parallel to the ordinate axis $a_{0}^{2}$ and cutting the resonance curve at a point $M$, if we go from the region $D W<0(>0)$ to the region $D W>0(<0)$ then point $M$ corresponds to the stable (unstable) oscillation. Therefore, basing on the sign distribution of the functions $D$ and $W$ in the $P$-plane, we can identify the stable and unstable branches of the resonance curve.

\section{THE CASE OF ARBITRARY PARAMETERS. REGULAR AND CHAOTIC SOLUTIONS}

Les us go back to the equation (2.1), ignoring the assumption on the smallness of the parameters, i.e. we will consider the following differential equation

$$
\ddot{x}+\omega^{2} x=\Delta x-\gamma x^{3}+h\left(1-k x^{2}\right) \dot{x}+2 p x \cos 2 \omega t+e \cos (\omega t+\sigma) .
$$

We fix the parameters: $\omega=0.83, \Delta=0.01, \gamma=1, h=1, k=0.6, p=0.001, \sigma=0$ and use $e$ as a control parameter. With different values of $e$, solutions of the equation (3.1) can be regular or chaotic. To identify the regular or chaotic character of a solution, we can use various methods, such as consideration of the sign of the largest Lyapunov exponent, or building the Poincaré sections $[4,10]$. To construct a Poincaré section of an orbit, we use the period $T=\frac{2 \pi}{\omega}$ of the external excitation force. Then, the Poincaré section acts like a stroboscope, freezing the components of the motion commensurate with the period $T$. If we have a collection of $n$ discrete points on the Poincaré section, the corresponding motion is periodic with the period $n T$. For example, for $e=5.09$, the Poincaré section consists of three points (Fig. 5a), the motion is periodic with the period 3T; for $e=5.116$, the Poincaré section consists of six points (Fig. 5b), the motion is periodic with the period $6 T$. When the Poincaré section does not consist of finite number of discrete points, the motion is aperiodic, it may be chaotic (Fig. 6).
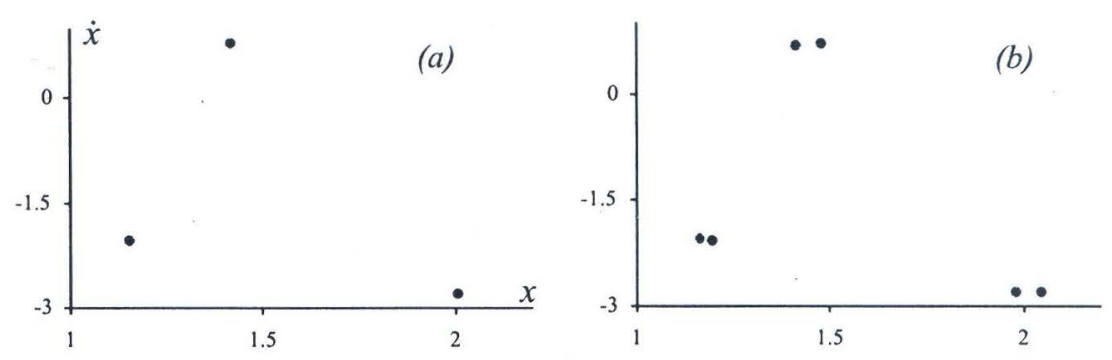

Fig. 5. Poincaré section: $e=5.09$ (a), $e=5.116$ (b) 

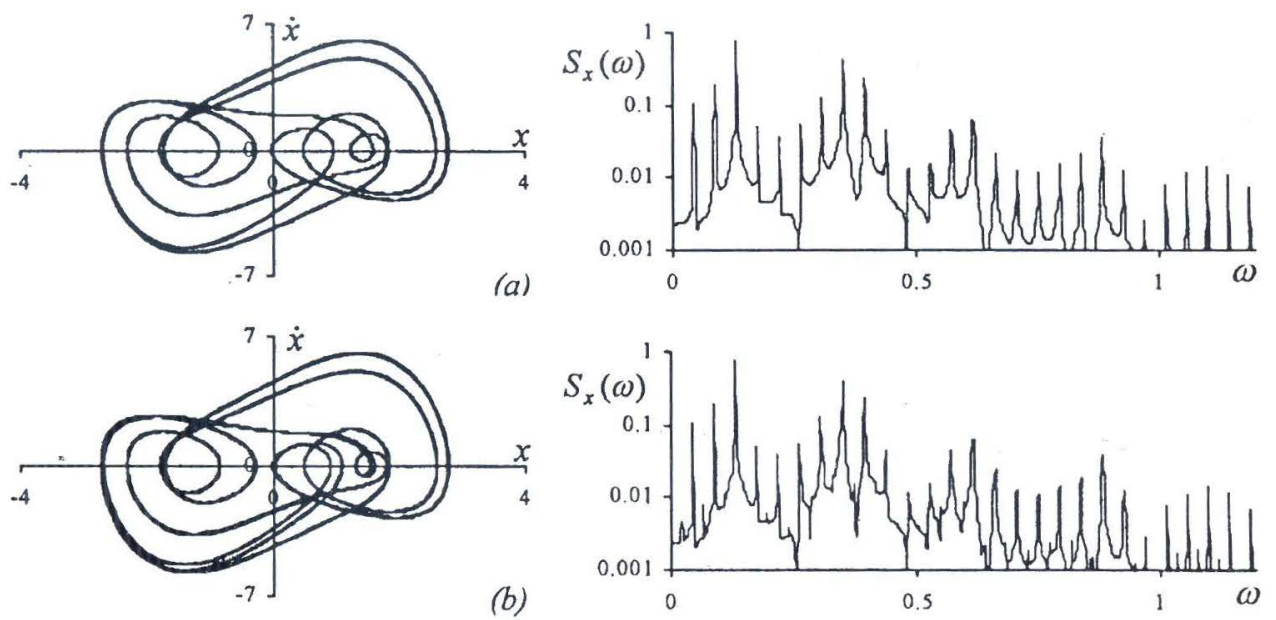

Fig. 6. Periodic attractors and associated power spectra: $e=5.09$ (a), $e=5.116$ (b)

The periodic attractors and the corresponding power spectrums realized at $e=5.09$ and $e=5.116$ are illustrated in Fig. 6a, Fig. 6b. The aperiodic attractor and its power spectrum realized at $e=5.15$ are illustrated in Fig. 8. In this case the power spectrum has a continuous broadband character. The Poincaré section has a distinctive form shown in Fig. 7, it consists of about 8000 points after the transition decays (the first 500 periods)

To verify that the motion realized at $e=5.15$ is chaotic, we need to show the sensitivity to initial conditions on this attractor. We choose two points separated by $d_{0}=10^{-7}$ close to the attractor and examine evolutions initiated from them. Fig. 9 illustrates the variation of the separation $d$ with time $t$. The exponential growth of separation for $20<t<300$ is clearly noticeable. The separation saturates at the size of the attractor for $t>300$. Therefore, there is a positive Liapunov exponent associated with the chaotic orbit at $e=5.15$. The evaluated largest Lyapunov exponent is $\lambda \approx 0.062>0$ (Its calculation will be mentioned below).
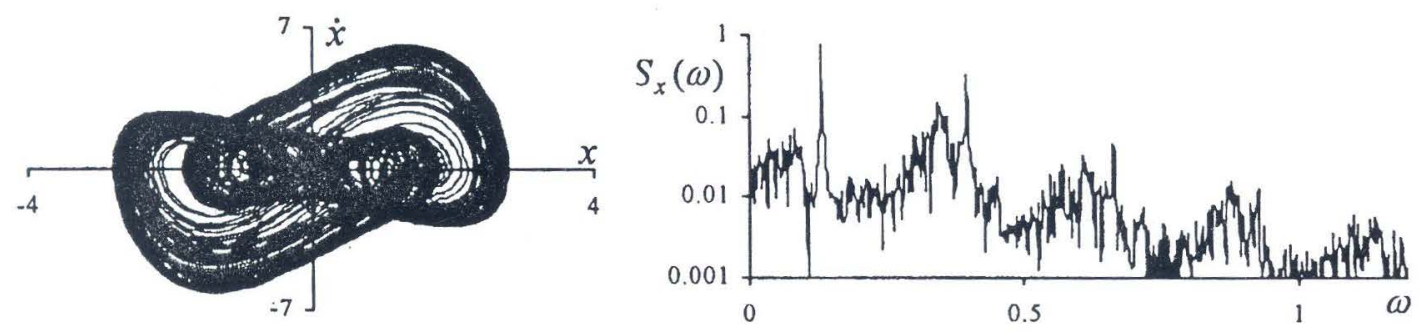

Fig. 7. Chaotic attractor and associated power spectra at $e=5.15$ 


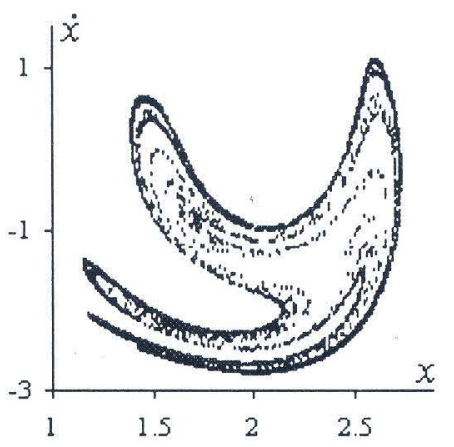

Fig. 8. Poincaré section realized at $e=5.15$

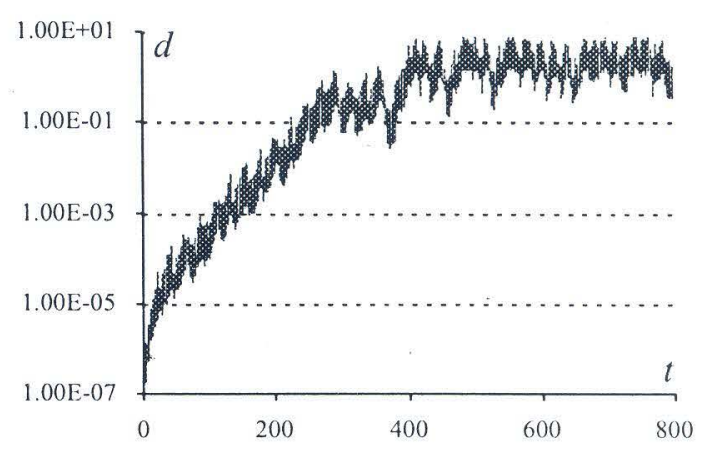

Fig. 9. Sensitivity to initial condition at $e=5.15$

The evaluation of the largest Liapunov exponent

To evaluate the largest Lyapunov exponent in the case $\omega=0.83, \Delta=0.01, \gamma=1$, $h=1, k=0.6, p=0.001, \sigma=0$ and $e=5.15$, we represent the equation (3.1) in the form

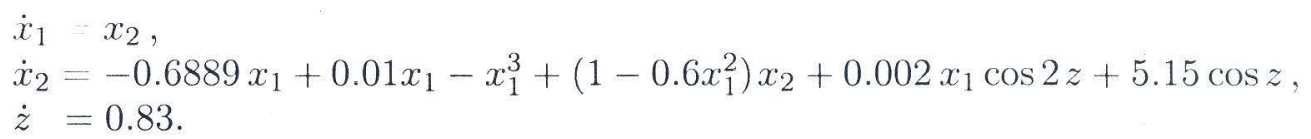

Let $u=\left(x_{1}, x_{2}, z\right)$ is a three dimensional vector and $u^{*}=u^{*}\left(t, u_{0}\right)$ is a reference trajectory of the system (3.2), where $u_{0}$ is the initial condition. The variational equation corresponding to this reference trajectory is

$$
\dot{\eta}=A \eta,
$$

where $\eta=u-u^{*}$ and the matrix $A$ depends on $u^{*}$

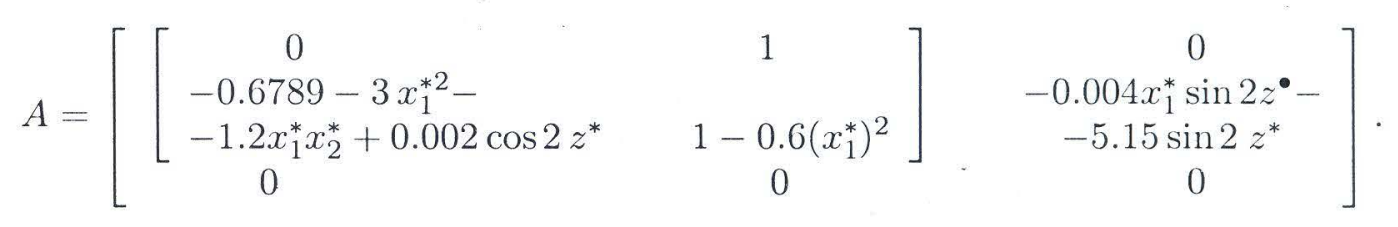

The time evolution of the Lyapunov exponent is presented in Fig. 10. The largest Lyapunov exponent is a positive number $\lambda \approx 0.062$, which shows the chaotic character of the motion of the system (3.2). This means that two trajectories starting closely one to another in the phase space will move exponentially away from cach other for small times on the average

$$
d(t)=d_{0} 2^{\lambda t},
$$

where $d_{0}$ is the initial distance between two adjacent starting points at $t=t_{0}$ and $d$ is the distance between two these points at the moment $t$. We return again to Fig. 9, it shows how the distance $d$ between evolutions initiated from two points separated by $d_{0}=10^{-7}$ varies with time. The separation grows exponentially in the range $20<t<300$ before leveling off at the size of the attractor. 


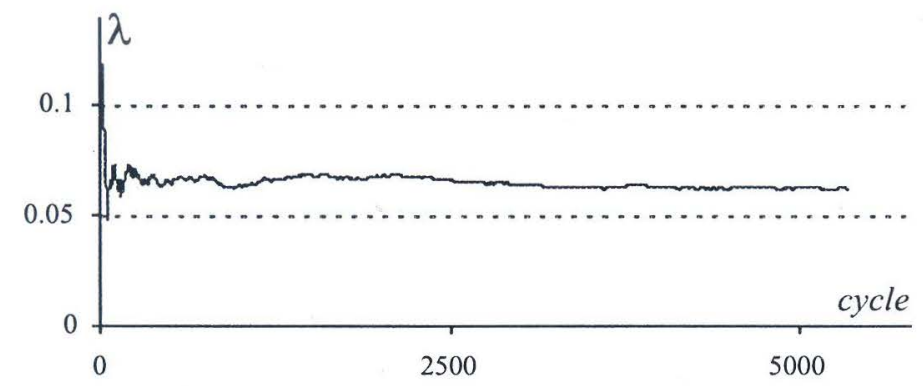

Fig. 10. Time evolution of the largest Lyapunov exponent (one cycle $=2 \pi / \omega, \omega=0.83$ )

\section{CONCLUSION}

The first section of the paper shows how the asymptotic method created by Krylov, Bogoliubov and Mitropolskii is effective in solving a complicated nonlinear problem. Fig. 2 presents different resonance curves in "linear" case $\gamma=0$. There exists a special returning point $I_{*}$ on the resonance curves. In the "nonlinear case" $\gamma \neq 0$, the resonance curve leans toward to the right for $\gamma>0$ (Fig. 3b), and to the left for $\gamma<0$ (Fig. 3a), which is common for nonlinear Duffing's systems.

In the second section it is seen that chaotic phenomenon occurs in a deterministic system described by (3.1). Poincaré section, chaotic attractor and associated power spectra of the nonlinear oscillator (3.1) have been found. The Fortran and Matlab softwares were used for calculating data and building the graphs.

This work is completed with the financial support of the Council for Natural Science of Vietnam.

\section{REFERENCES}

1. Yu. A. Mitropolskii, Nguyen Van Dao, Applied Asymptotic Methods in Nonlinear Oscillations, Kluwer Publisher, 1997, 341p.

2. Yu. A. Mitropolskii, Nguyen Van Dao, Lectures on asymptotic methods of nonlinear dynamics, Vietnam national University Publishing House, Hanoi, 2003, 503p.

3. Nguyen Van Dao, Nguyen Van Dinh, Interaction between nonlinear oscillating systems, Vietnam national University Publishing House, Hanoi, 1999, 358p.

4. A. Nayfeh, Balakuma Balachandran, Applied Nonlinear Dynamics, A Wiley-Interscience Publication, 1995. John Wiley \& Sons, Inc., 685p.

5. Y. Ueda, The Road to Chaos, Aerial Press, Inc.1992.

6. T. Kapitaniak, W. H. Steeb, Transition to chaos in a generalized Van der Pol's equation, Journal of Sound and Vibration 143 (1) (1990) 143 167-170.

7. M. Lakshmanan, S. Rajasekar, Nonlinear Dynamics, Springer, 2003.

8. G. L. Baker, J.'P. Gollub, Chaotic Dynamics, An introduction, Cambridge University Press, 1990.

9. Francis C Moon, Chaotic Vibration, John wiley, 1996.

10. Kathleen T. Alligood, Tim D. Sauer, Jame A. Yorke. Chaos, An Introduction to Dynamical Systems, Springer Verlag, New York, Inc. 1997.

11. Nguyen Van Dao, Stability of Dynamic Systems, Vietnam National University Publishing House, Hanoi, 1998.

12. Nguyen Van Dao, Tran Kim Chi, Nguyen Dung, An introduction to nonlinear dynamics \& Chaos, Vietnam National University Publishing House, Hanoi, 2005, 201p. 


\section{CHẤN TƯ VAN DER POL DƯỚI CÁC KÍCH ĐộNG CƯỠNG BỨC VÀ THAM SỐ}

Bài này trình bày các nghiên cứu về chấn tử Van der Pol chịu các kích động tham số và cưỡng bức. Đã nghiên cứu trường hợp tựa tuyến tính khi hệ chứa một tham số bé và trường hợp tổng quát (không có giả thiết bé về số hạng phi tuyến và nhiễu). Trong trường hợp đầu, các phương trình xấp xỉ thứ nhất thu được bằng phương pháp Krylov-Bogoliubov-Mitropolskii, đã xây dựng các trung bình, đã nghiên cứu quan hệ tần số - biên độ và các đường cong cộng hường, và về sự ôn định của hệ. Trong trường hợp thứ hai, đã chỉ ra khá năng xuất hiện hỗn độn trong các hệ tất định thuộc dạng chấn tứ này. 